\title{
Estimativa da ÁRea Foliar de Typha latifolia Usando Dimensões LINEARES DO LIMBO FOLIAR ${ }^{1}$
}

\author{
Leaf Area Estimation in Typha latifolia Using Leaf Blade Linear Dimensions \\ BIANCO, S. ${ }^{2}$, PITELLI, R.A. ${ }^{2}$ e PITELLI, A.M.C.M. ${ }^{4}$
}

\begin{abstract}
RESUMO - Com o objetivo de obter uma equação que, através de parâmetros lineares dimensionais das folhas, permita a estimativa da área foliar de Typha latifolia, estudaram-se relações entre a área foliar real (Sf) e parâmetros dimensionais do limbo foliar, como o comprimento ao longo da nervura principal (C) e a largura máxima (L), perpendicular à nervura principal. As equações lineares simples, exponenciais e geométricas obtidas podem ser usadas para estimação da área foliar da taboa. Do ponto de vista prático, sugere-se optar pela equação linear simples que envolve o produto C x L, usando-se a equação de regressão $\mathrm{Sf}=0,9651 \times(\mathrm{C} \times \mathrm{L})$, que equivale a tomar $96,51 \%$ do produto entre o comprimento ao longo da nervura principal e a largura máxima, com um coeficiente de determinação de 0,9411.
\end{abstract}

Palavras-chave: planta daninha, área foliar, taboa.

\begin{abstract}
This study aimed to obtain a mathematical equation to estimate the leaf area of Typha latifolia, using leaf blade linear dimensions. Correlation studies were carried out involving real leaf area and leaf length $(C)$, maximum leaf width $(L)$ and $C * L$. The linear and geometric equations involving the parameter $C$ provided good estimates of leaf area. From a practical viewpoint, it is suggested to choose the simple linear equation of the regression model using the $C^{*} L$ parameter and taking the linear coefficient equal to zero, since it shows the smallest Error of Sum of Squares. Thus, an estimation of Typha latifolia leaf area can be obtained using the equation $S f=0,9651(C * L)$, with a determination coefficient of $R^{2}=0,9411$.
\end{abstract}

Key words: weeds, leaf area, "taboa".

\section{INTRODUÇÃO}

Typha latifolia, vulgarmente conhecida por taboa, é uma planta pertencente à família Typhaceae, originária da América do Sul. É uma planta daninha aquática muito freqüente em margens de lagos, lagoas ou represas, canais de drenagem e baixadas pantanosas em geral. É bastante vigorosa, chegando a produzir $7.000 \mathrm{~kg}$ de rizomas por hectare. Seus rizomas são comestiveis, possuindo valor protéico igual ao do milho e de carboidratos igual ao da batata. Plantas de Typha absorvem metais pesados, inclusive o cobre, podendo contribuir para o saneamento ambiental (Kissmann, 1997).

Sob o ponto de vista negativo, quando em povoamentos densos, essas plantas provocam desequilibrio, tornando-se infestantes em açudes e várzeas úmidas, diminuindo ou impedindo seu aproveitamento adequado. Outro fator negativo é que nos povoamentos de taboas existem excelentes condições para a reprodução de mosquitos.

Considerando-se a importância dessa planta, há grande necessidade de estudos básicos envolvendo aspectos relacionados a

Recebido para publicação em 24.3.2003 e na forma revisada em 11.8.2003.

2 Prof. Dr., Departamento de Biologia Aplicada à Agropecuária - FCAV-UNESP, Jaboticabal-SP; ${ }^{3}$ Prof. Titular. Departamento de Biologia Aplicada à Agropecuária - FCAV-UNESP, Jaboticabal-SP; ${ }^{4}$ Acadêmico em Agronomia, FCAV-UNESP, JaboticabalSP. 
reprodução, crescimento, desenvolvimento, exigências em nutrientes, respostas aos sistemas de controle e outros. Na maioria desses estudos, o conhecimento da área foliar é fundamental, por ser talvez o mais importante parâmetro na avaliação do crescimento vegetal. É uma das características mais dificeis de serem mensuradas, porque normalmente requer equipamentos caros ou técnicas destrutivas, como comentam Bianco et al. (1983). Existem vários métodos para se medir a área foliar, a maioria com boa precisão. Marshall (1968) os classificou em destrutivos e não-destrutivos, diretos ou indiretos. A importância de se utilizar um método não-destrutivo é que ele permite acompanhar o crescimento e a expansão foliar da mesma planta até o final do ciclo ou do ensaio, além de ser rápido e preciso. Assim, a área foliar pode ser estimada utilizando-se parâmetros dimensionais de folhas, os quais apresentam boas correlações com a superficie foliar. Um dos métodos não-destrutivos mais utilizados é a estimativa da área foliar por meio de equações de regressão entre a área foliar real (Sf) e parâmetros dimensionais lineares das folhas. Este método já foi utilizado com sucesso para inúmeras plantas cultivadas como abóbora (Silva et al., 1998), videira em cultivar Niagara Rosada (Pedro Junior et al., 1986), entre outras - e plantas daninhas, como Wissadula subpeltata (Bianco et al., 1983), Senna obtusifolia (Peressin et al., 1984), Amaranthus retroflexus (Bianco et al., 1995), Richardia brasiliensis (Rosseto et al., 1997), Solanum americanum (Tofoli et al., 1998b), Cissampelos glaberrima (Bianco et al., 2002), entre outras.

O presente trabalho teve como objetivo determinar uma relação ou equação adequada para estimar a área foliar da taboa Typha angustifolia, por intermédio de medidas lineares de seus limbos foliares.

\section{MATERIAL E MÉTODOS}

Foram coletados 200 limbos foliares de T. latifolia sujeitos às mais diversas condições ecológicas em que a espécie pode ocorrer, considerando-se todas as folhas das plantas, desde que não apresentassem deformações oriundas de fatores externos, como pragas, moléstias e granizo. Na fase de coleta dos dados, foram realizadas rápidas excursões ao campo, coletando-se de 10 a 20 folhas de diferentes plantas, as quais eram levadas ao laboratório, para determinação do comprimento do limbo foliar ao longo da nervura principal (C) e da largura máxima do limbo foliar (L) perpendicular à nervura principal. A seguir, suas áreas foliares reais (Sf) foram determinadas com a utilização do aparelho Portable Area Meter Licor, modelo L1 - 3000.

Para escolha de uma equação que possa representar a área foliar em função das dimensões foliares, procedeu-se a estudos de regressão, utilizando as seguintes equações: linear $\mathrm{Y}=\mathrm{a}+\mathrm{bx}$; linear pela origem $\mathrm{Y}=\mathrm{bx}$, geométrica $\mathrm{Y}=a x^{\mathrm{b}}$ e exponencial $\mathrm{Y}=a b^{\mathrm{x}}$. $\mathrm{O}$ valor Y estima a área foliar do limbo foliar em função de $X$, cujos valores podem ser o comprimento $(\mathrm{C})$, a largura $(\mathrm{L})$ ou o produto $(\mathrm{C}$ $x \mathrm{~L})$. No caso de $\mathrm{X}$ igual a $(\mathrm{C} \times \mathrm{L})$, estimou-se também a equação linear passando pela origem, o que praticamente significa supor que a área é proporcional a um retângulo $(\mathrm{C} \times \mathrm{L})$. Todas as equações utilizadas são lineares ou linearizáveis por transformação, de modo que os ajustes foram feitos a partir de retas. Para realizar as comparações entre os modelos, foram obtidas as somas de quadrados das diferenças entre os valores observados e os preditos pelos modelos, denominando isso de soma de quadrados do resíduo. No caso dos modelos com transformação (geométrica e exponencial), foi feita a volta para escala original e, depois disso, obtidas as referidas somas de quadrados do resíduo. A melhor equação é a que apresenta a menor soma de quadrados do resíduo na escala real (sem transformação).

Os coeficientes de determinação são os obtidos com as variáveis de trabalho $\mathrm{X} \mathrm{e} \mathrm{Y,} \mathrm{no}$ caso linear; logaritmo de $\mathrm{Y}$ e logaritmo de $\mathrm{X}$, no caso geométrico; e logaritmo de $\mathrm{Y}$ e $\mathrm{X}$, no caso exponencial. O número de graus de liberdade é o número de folhas analisadas, menos o número de parâmetros estimados para cada modelo. Para se testar o acréscimo de soma de quadrados do resíduo do modelo passando pela origem, em relação ao modelo com intercepto, utilizou-se o teste $\mathrm{F}$ condicional: $\mathrm{F}=$ (SQRes. $(0,0)$ - SQRes. CL)/SQRes. CL/GL), com 1 e 2 GL (graus de liberdade), em que GL é o número de folhas menos 2 (Mead \& Curnow, 1983; Neter \& Wasserman, 1974). 


\section{RESULTADOS E DISCUSSÃO}

Os resultados de regressão efetuados, relacionando a área foliar real (Sf) e as medidas lineares de comprimento (C), largura (L) e o produto do comprimento pela largura da folha $(\mathrm{C} \times \mathrm{L})$, estão na Tabela 1 . Todas as equações apresentadas permitiram obter estimativas satisfatórias da área foliar de T. latifolia. Nas equações linear, geométrica e exponencial envolvendo apenas o componente largura da folha foi obtido coeficiente de determinação abaixo de 0,60 , indicando pouca precisão nas estimativas da área foliar. Nas demais equações envolvendo o comprimento da folha e o produto do comprimento pela largura foram obtidos coeficientes de determinação acima de 0,90, indicando que $90,00 \%$ das variações observadas na área foliar foram explicadas pelas equações obtidas. As equações que representam o produto entre o comprimento e a largura, passando ou não pela origem, não mostraram diferenças significativas quando comparadas entre si; isso era esperado, visto que a retirada de uma constante não afeta o comportamento dos dados (Neter \& Wasserman, 1974).

Os valores do comprimento (C) das folhas variaram de 38,5 a 194,0 cm, com valores médios de $126,19 \mathrm{~cm}$, enquanto a largura (L) máxima das folhas variou de 1,0 a $2,2 \mathrm{~cm}$, com valores médios de $1,83 \mathrm{~cm}$. Para a área foliar real, os valores variaram entre 40,88 e $392,37 \mathrm{~cm}^{2}$ e a média foi de $228,78 \mathrm{~cm}^{2}$ (Tabela 2).
A distribuição percentual dos 200 limbos foliares da taboa, em relação às faixas de tamanho, encontra-se na Tabela 3. Observase que $77,0 \%$ da área foliar está relacionada com folhas variando de 150,10 a $350,00 \mathrm{~cm}^{2}$ de área, indicando que esta planta daninha possui a maioria de suas folhas de tamanho grande, no que diz respeito principalmente ao comprimento das folhas.

Os maiores valores do coeficiente de determinação e os menores valores da soma de quadrados do resíduo foram observados para as regressões lineares simples entre a área foliar real e o produto do comprimento pela largura da folha, mostrando serem as equações que permitem obter estimativas mais acuradas da área foliar da taboa (Tabela 1). Nota-se que estas equações apresentaram estimativas do coeficiente de determinação de 0,9411, indicando que, da variabilidade total existente na área foliar, $94,11 \%$ pode ser explicada pela

Tabela 2 - Valores máximos, mínimos e médios do comprimento ao longo da nervura central, largura e área foliar de 200 limbos de Typha latifolia. FCAV-UNESP. Jaboticabal-SP, 2003

\begin{tabular}{|l|c|c|c|}
\hline \multicolumn{1}{|c|}{ Característica } & Maior valor & Menor valor & Média \\
\hline Comprimento $(\mathrm{cm})$ & 194,00 & 38,50 & 126,19 \\
\hline Largura máxima $(\mathrm{cm})$ & 2,20 & 1,00 & 1,83 \\
\hline Área foliar $\left(\mathrm{cm}^{2}\right)$ & 392,37 & 40,88 & 228,78 \\
\hline
\end{tabular}

Tabela 1 - Equações de regressão estimadas, coeficientes de determinação, graus de liberdade e somas de quadrados de desvios da regressão da área foliar em função das medidas lineares do limbo foliar de Typha latifolia. FCAVUNESP. Jaboticabal-SP, 2003

\begin{tabular}{|l|c|c|c|c|c|}
\hline $\mathrm{X}^{1}{ }^{\prime}$ & Tipo de Equação & $\begin{array}{c}\text { Coeficiente de } \\
\text { Determinação }\end{array}$ & GL & $\begin{array}{c}\text { S. Q. resíduo } \\
\text { (na escala original) }\end{array}$ & Equação Estimada (Sf) \\
\hline C & linear & 0,9231 & 198 & $198.254,8830$ & $-45,2445+2,180 \mathrm{C}$ \\
\hline L & linear & 0,6031 & 198 & $852.462,3554$ & $-99,4424+178,403 \mathrm{~L}$ \\
\hline CL & linear & 0,9411 & 198 & $153.272,3558$ & $9,1568+0,9306 \mathrm{CL}$ \\
\hline CL(0,0) & linear & 0,9411 & 199 & $155.094,6340$ & $0,9651 \times \mathrm{CL}$ \\
\hline C & geométrica & 0,8449 & 198 & $201.598,6897$ & $0,4602 \times \mathrm{C}^{1,2798}$ \\
\hline L & geométrica & 0,5916 & 198 & $875.764,7130$ & $80,4848 \times \mathrm{L}^{1,6109}$ \\
\hline C & exponencial & 0,5916 & 198 & $275.079,3743$ & $48,5148 \times 1,0117^{\mathrm{C}}$ \\
\hline L & exponencial & 0,5942 & 198 & $878.384,7614$ & $37,0870 \times 2,5700^{\mathrm{L}}$ \\
\hline
\end{tabular}

${ }^{1 /}$ medidas lineares: comprimento (C) e largura (L). 
regressão linear. A equação linear simples com a reta passando pela origem é a mais recomendada, pois não altera expressivamente a soma de quadrados do resíduo e é de mais fácil utilização do ponto de vista prático. Assim, a estimativa da área foliar da taboa pode ser feita pela equação $\mathrm{Sf}=0,9651 \times(\mathrm{C} \times \mathrm{L})$, ou seja, corresponde a $96,51 \%$ do produto entre o comprimento e a largura máxima da folha, ou $96,51 \%$ da área dada pelo comprimento $x$ largura (Figura 1).

Tabela 3 - Distribuição percentual de 200 limbos foliares de Typha latifolia em relação às faixas de tamanho. FCAV - UNESP. Jaboticabal-SP, 2003

\begin{tabular}{|c|c|}
\hline Tamanho $\left(\mathrm{cm}^{2}\right)$ & $(\%)$ \\
\hline$[0,00-50,00]$ & 1,0 \\
\hline$[50,01-100,00]$ & 6,5 \\
\hline$[100,01-150,00]$ & 9,5 \\
\hline$[150,01-200,00]$ & 21,5 \\
\hline$[200,01-250,00]$ & 16,5 \\
\hline$[205,01-300,00]$ & 20,0 \\
\hline$[300,01-350,00]$ & 19,0 \\
\hline$[350,01-400,00]$ & 6,0 \\
\hline
\end{tabular}

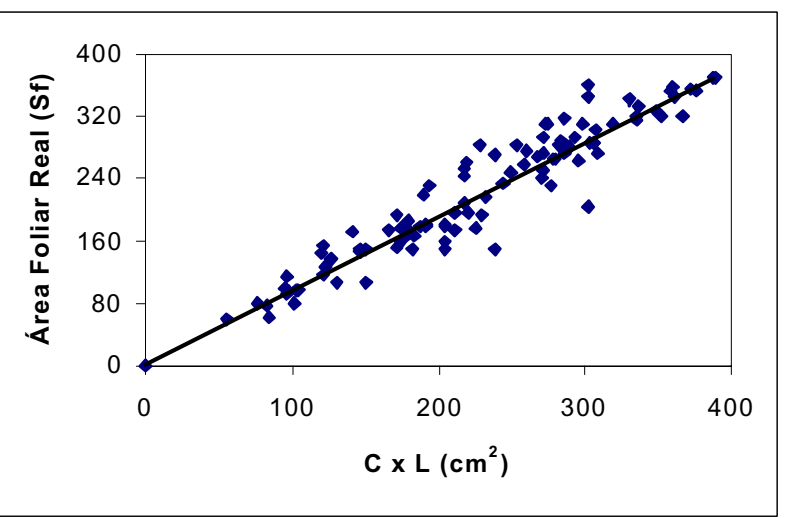

Figura 1 - Representação gráfica da área foliar de Typha latifolia e da equação de regressão indicada para estimativa da área foliar da planta daninha, em função do produto do comprimento (C) pela largura (L) máxima do limbo foliar.

Houve pequena dispersão dos dados em relação à reta obtida, sugerindo que a equação $\mathrm{Sf}=0,9651 \times(\mathrm{C} \times \mathrm{L})$ pode representar a área foliar real muito satisfatoriamente (Figura 1). $O$ valor obtido é inferior aos observados para Amaranthus retroflexus (Bianco et al., 1995), Richardia brasiliensis (Rosseto et al., 1997), Nicandra physaloides (Bianco et al., 1996), Raphanus raphanistrum (Tofoli et al., 1998a), Solanum americanum (Tofoli et al., 1998b) e Portulaca oleracea (Pedrinho Junior et al., 2000).

Os resultados encontrados no presente trabalho permitem concluir que as equações obtidas podem ser utilizadas para estimar a área foliar de $T$. latifolia e que, do ponto de vista prático, a área foliar é estimada utilizando-se a equação $\mathrm{Sf}=0,9651 \times(\mathrm{C} \times \mathrm{L})$.

\section{LITERATURA CITADA}

BIANCO, S.; PITELli, R. A.; PERECIN, D. Métodos para estimativa da área foliar de plantas daninhas. 2. Wissadula subpeltata (Kuntze) Fries. Planta Daninha, v. 6, n. 1, p. 2124, 1983.

BIANCO, S. et al. Estimativa de área foliar de plantas daninhas. XIII - Amaranthus retroflexus L. Ecossistema, v. 20, p. 5-9, 1995.

BIANCO, S.; PITELli, R. A.; CARVALHO, L. B. Estimativa da área foliar de Typha angustifolia L. usando dimensões lineares do limbo foliar. Planta Daninha, v. 20, n. 3, p. 353-356, 2002.

BIANCO, S.; PITELLI, R. A.; SILVA, R. C. Estimativa da área foliar de plantas daninhas. XIV. Nicandra physaloides (L.) Pers. Cultura Agron., v. 5, n. 1, p. 33-38, 1996.

BIANCO, S. et al. Estimativa da área foliar de plantas daninhas: Brachiaria decumbens Stapf. e Brachiaria brizantha (Hochst.) Stapf. Planta Daninha, Londrina, v. 18 , n. 1 , p. $79-83,2000$.

KISSMANN, K. G. Plantas infestantes e nocivas. 2.ed. São Paulo: BASF, 1997-T.1. 825. p. 1997.

MARSHALL, J. K. Methods of leaf area measurement of large and small leaf samples. Photosynthetica, v. 2, p. 4147, 1968.

MEAD, R.; CURNOW, R. N. Statistical methods in agriculture and experimental biology. New York: Chapman and Hall, 1983. 335 p.

NETER, J.; WASSERMAN, W. Applied models. Regressions, analysis of variance and experimental designs. Illinois: Rechard D. Irwin, 1974. 842 p.

PEDRO JUNIOR, M. J.; RIBEIRO, I. J. A.; MARTINS, F. P. Determinação da área foliar em videira cultivar Niagara Rosada. Bragantia, v. 45, n. 1, p. 199-204, 1986. 
PEDRINHO JUNIOR, A. F. F. et al. Estimativa da área foliar de plantas daninhas: Portulaca oleracea L.

Ecossistema, v. 25, p. 86-88, 2000.

PERESSIN, V. A.; PITELLI, R. A.; PERECIN, D. Métodos para estimativa da área foliar de plantas daninhas. 4. Cassia tora L. Planta Daninha, v. 7, n. 2, p. 48-52, 1984.

ROSSETO, R. R.; PITELLI, R. L. C. M.; PITELLI, R. A.

Estimativa da área foliar de plantas daninhas: poaia-branca. Planta Daninha, v. 15, n. 1, p. 25-29, 1997.
SILVA, N. F. et al. Modelos para estimar a área foliar de abóbora por meio de medidas lineares. R. Ceres, v. 45, n. 259, p. 287-291, 1998.

TOFOLI, G. R.; BIANCO, S.; PAVANI, M. C. M. D. Estimativa da área foliar de Solanum americanum Mill. Planta Daninha, v. 16, n. 2, p. 149-152, 1998 b.

TOFOLI, G. R. et al. Estimativa da área foliar de plantas daninhas (Raphanus raphanistrum L.). Ecossistema, v. 23, p. 5-7, 1998a. 